\title{
Numerical Stability and Accuracy of the Scaled Boundary Finite Element Method in Engineering Applications
} Miao Li ${ }^{\mathrm{a}}$, Yong Zhang ${ }^{\mathrm{b}}$, Hong Zhang ${ }^{\mathrm{a}, *}$ and Hong Guan ${ }^{\mathrm{a}}$

a Griffith School of Engineering, Griffith University, $Q L D, 4222$, Australia

b Institute of Nuclear Energy Safety Technology, Chinese Academy of Sciences, Hefei, Anhui, 230031, China

*Corresponding author. Tel: +61755529015. Email address: hong.zhang@griffith.edu.au

\begin{abstract}
The Scaled Boundary Finite Element Method (SBFEM) is a semi-analytical computational method initially developed in the 1990s. It has been widely applied in the fields of solid mechanics, as well as oceanic, geotechnical, hydraulic, electromagnetic, and acoustic engineering problems. Most of the published work on SBFEM has so far emphasised on its theoretical development and practical applications, and no explicit discussion on the numerical stability and accuracy of the SBFEM solution has been systematically documented so far. In order for a more reliable application in engineering practice, the inherent numerical problems associated with SBFEM solution procedures require thorough analysis in terms of its causes and the corresponding remedies. This study investigates the numerical performance of SBFEM with respect to matrix manipulation techniques and matrix properties. Some illustrative examples are employed to identify reasons for possible numerical difficulties, and corresponding solution schemes are also discussed to overcome these problems.
\end{abstract}

Key words: SBFEM; numerical stability and accuracy; matrix decomposition; nondimensionalisation; engineering application

\section{Introduction}

The development of SBFEM can be dated back to mid-1990s [25]. It was initially termed as the Consistent Infinitesimal Finite-Element Cell Method, and was renamed as the Scaled Boundary Finite Element Method when the concept of solving problems was 
better understood. Since then, SBFEM has been utilised in various engineering fields with rapid recognition and acknowledgment. Apart from the wave propagation problem within the framework of dynamic unbounded medium-structure interaction, from which the concept of SBFEM was originally derived, SBFEM has been employed in fracture mechanics $[28,29,30]$ by taking advantage of its capability to accurately capture the stress intensification around the crack tips. It has also been applied to solve wave diffraction problems around breakwaters and caissons by many researchers [10, 11, 23, 24]. Subsequently, SBFEM has been reformulated in computational electromagnetics to address waveguide eigenproblems [13], extending its application to a new area.

One of the most significant concerns when assessing SBFEM's practical applicability, which is the same as other numerical methods, lies in the reliability of its solution, more specifically, the numerical stability and accuracy of its calculations. The original partial differential equations (PDEs) governing the physical problem, through the scaled boundary coordinate transformation and the weighted residual technique, is rewritten in the matrix-form of ordinary differential equations (ODEs), i.e., the scaled boundary finite element equation. The term 'matrix' refers to the coefficient matrices of the equation, which are calculated from the discretisation information of the domain boundary and are in the form of matrices. These coefficient matrices are used to formulate a Hamiltonian matrix, of which a matrix-decomposition is to be performed. The level of accuracy of the Hamiltonian matrix decomposition is a prerequisite for a valid SBFEM calculation. On the other hand, SBFEM is essentially vulnerable to the unavoidable rounding error associated with floating-point arithmetic, especially when the magnitudes of matrix entries calculated from input parameters differ significantly over a vast range. The rounding error can intensify over a sequence of matrix manipulations, especially matrix inversions, to such an unmanageable extent that it renders the SBFEM calculation meaningless.

Most of the literature in this area has focused on the theoretical development of SBFEM in terms of deriving its conceptual framework [6, 20,21, 22, 26, 27], and the technical issues in relation to the solution algorithms of the scaled boundary finite element equation $[1,2,12,17,18]$. No explicit emphasis has been given to the numerical stability 
and accuracy of the SBFEM solution, which leads to a discussion on its practical applicability. Filling this research gap is the motivation of this study in which the numerical credibility of SBFEM is explored, the technical reasons for the potential instability and inaccuracy are detected, and the corresponding solution schemes to overcome these problems are proposed.

\section{Basic formulations of SBFEM}

The concept of SBFEM originates from two robust numerical methods, i.e., the Finite Element Method (FEM) and the Boundary Element Method. By scaling the discretised boundary of the study domain with respect to a centre either outwards to address an unbounded domain, or inwards for a bounded domain, SBFEM describes the problem in question by using a radial coordinate and two circumferential coordinates. This reduces the spatial dimension of the problem by one in the solution process, as in the Boundary Element Method. The discretisation and assembly concepts are inherited from FEM, however, they are only applied on the boundary, which significantly minimises the discretisation effort and leads to substantially reduced degrees of freedom.

Detailed and systematic descriptions of key technical derivations of SBFEM and its solution schemes are abundantly documented and hence will not be duplicated. However a three-dimensional illustration of a bounded elastic problem is outlined herein to introduce some key equations for later reference.

The scaled boundary coordinate system $(\xi, \eta, \zeta)$, with $\xi$ denoting the radial coordinate and $\eta$ and $\zeta$ for the circumferential coordinates, is illustrated in Figure 1. It is interrelated to the Cartesian coordinate system $(\hat{x}, \hat{y}, \hat{z})$ by the mapping function $[N(\eta, \zeta)]$ as:

$$
\begin{aligned}
& \hat{x}(\xi, \eta, \zeta)=\xi[N(\eta, \zeta)]\{x\}+x_{0} \\
& \hat{y}(\xi, \eta, \zeta)=\xi[N(\eta, \zeta)]\{y\}+y_{0} \\
& \hat{z}(\xi, \eta, \zeta)=\xi[N(\eta, \zeta)]\{z\}+z_{0}
\end{aligned}
$$

where $(\{x\},\{y\},\{z\})$ represents a nodal point on the discretised boundary; $\left(x_{0}, y_{0}, z_{0}\right)$ represents the scaling centre $O$ with respect to which the boundary is scaled. Note that as a convention in SBFEM, the coordinate of the Cartesian space is represented by $(\hat{x}, \hat{y}, \hat{z})$ and $(x, y, z)$ is reserved for the coordinates on the boundary. However, $x, y$ and $z$ are still 
used when indicating directions in the following discussions.

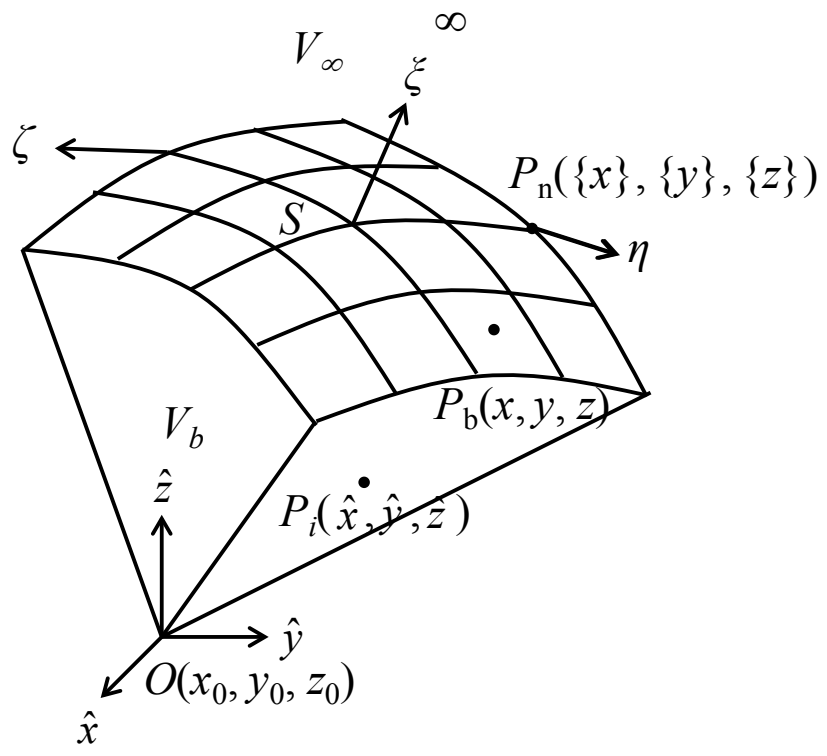

Figure 1. Definition of the scaled boundary coordinate system [20].

Equation (1), upon which the scaled boundary transformation is based, is the core of the SBFEM concept. The governing differential equations for elasto-dynamic problems are shown in equation (2), with $[L]$ representing the differential operator, $\{\sigma\}$ the stress amplitude, $\{\varepsilon\}$ the strain amplitude, $\{u\}$ the displacement amplitude, $[D]$ the elastic matrix, $\omega$ the excitation frequency, and $\rho$ the mass density:

$$
\begin{gathered}
{[L]^{T}\{\sigma\}+\omega^{2} \rho\{u\}=0} \\
\{\sigma\}=[D]\{\varepsilon\} \\
\{\varepsilon\}=[L]\{u\}
\end{gathered}
$$

Equation (2) is weakened along the discretised circumferential direction by employing either the weighted residual technique or the variational principle. Consequently, the scaled boundary finite element equation yields, and expressed in the nodal displacement function $\{u(\xi)\}$ as:

$$
\begin{aligned}
& {\left[E^{0}\right] \xi^{2}\{u(\xi)\}_{, \xi \xi}+\left(2\left[E^{0}\right]+\left[E^{1}\right]^{T}-\left[E^{1}\right]\right) \xi\{u(\xi)\}_{, \xi}+\left(\left[E^{1}\right]^{T}-\left[E^{2}\right]\right)\{u(\xi)\}} \\
& +\omega^{2}\left[M^{0}\right] \xi^{2}\{u(\xi)\}=0
\end{aligned}
$$

with the internal nodal force $\{q(\xi)\}$ written as: 


$$
\{q(\xi)\}=\left[E^{0}\right] \xi^{2}\{u(\xi)\}_{, \xi}+\left[E^{1}\right]^{T} \xi\{u(\xi)\}
$$

$\left[E^{0}\right],\left[E^{1}\right],\left[E^{2}\right]$ and $\left[M^{0}\right]$ are the coefficient matrices obtained by boundary discretisation and assemblage.

Equation (3) is termed as the scaled boundary finite element equation. It is a linear second-order matrix-form ordinary differential equation, the solution $\{u(\xi)\}$ of which represents the analytical variation of the nodal displacement in the radial direction. For elasto-static problems with $\omega=0$, equations (3) and (4) are formulated on the boundary where $\xi=1$. The nodal force $\{R\}$ - nodal displacement $\{u\}$ relationship is introduced in the following format:

$$
\{R\}=[K]\{u\}
$$

with $[K]$ representing the static stiffness matrix on the boundary. Equation (3) is solved by introducing the variable $\{X(\xi)\}$ to incorporate the nodal displacement function $\{u(\xi)\}$ and the nodal force function $\{q(\xi)\}$ as:

$$
\{X(\xi)\}=\left\{\begin{array}{c}
\xi^{0.5}\{u(\xi)\} \\
\xi^{-0.5}\{q(\xi)\}
\end{array}\right\}
$$

This results in first-order ordinary differential equations:

$$
\xi\{X(\xi)\}_{, \xi}=-[Z]\{X(\xi)\}
$$

with $[Z]$ being calculated by the coefficient matrices $\left[E^{0}\right],\left[E^{1}\right],\left[E^{2}\right]$ and the identity matrix $[I]$ as:

$$
[Z]=\left[\begin{array}{cc}
{\left[E^{0}\right]^{-1}\left[E^{1}\right]^{T}-0.5(s-2)[I]} & -\left[E^{0}\right]^{-1} \\
-\left[E^{2}\right]+\left[E^{1}\right]\left[E^{0}\right]^{-1}\left[E^{1}\right]^{T} & -\left[E^{1}\right]\left[E^{0}\right]^{-1}+0.5(s-2)[I]
\end{array}\right]
$$

with $s$ representing the spatial dimension of the study domain ( $s=2$ for two-dimensional problems and 3 for three-dimensional problems). For elasto-dynamic problems, the nodal displacement function $\{u(\xi)\}$ records the displacement variation history with respect to time. The nodal force $\{R\}$ - nodal displacement $\{u\}$ relationship is introduced as:

$$
\{R\}=[S(\omega)]\{u\}
$$

with $[S(\omega)]$ representing the dynamic stiffness matrix. With $\{R\}=\{q(\xi)\}$ at $\xi=1$ on the 5 
boundary, the scaled boundary finite element equation is rewritten using $[S(\omega)]$ as:

$$
\begin{aligned}
& \left([S(\omega)]-\left[E^{1}\right]\right)\left[E^{0}\right]^{-1}\left([S(\omega)]-\left[E^{1}\right]^{T}\right)-\left[E^{2}\right]+[S(\omega)] \\
& +\omega[S(\omega)]_{, \omega}+\omega^{2}\left[M^{0}\right]=0
\end{aligned}
$$

Equation (10) is a non-linear first-order matrix-form ODE. In this instance, the main objective is to solve the dynamic stiffness matrix $[S(\omega)]$ from equation (10) and back substitute to equation (9) to obtain the nodal degrees of freedom $\{u\}$.

Being formulated either in the nodal displacement function $\{u(\xi)\}$ or the dynamic stiffness matrix $[S(\omega)]$, once the nodal degrees of freedom $\{u\}$ is obtained, the solution of the entire domain can be calculated by specifying the scaled boundary coordinates $\xi, \eta$ and $\zeta$. The solution is exact in the radial direction and converges in the finite element sense in circumferential directions. The solution procedures described above can be illustrated by the flow chart shown in Figure 2. 


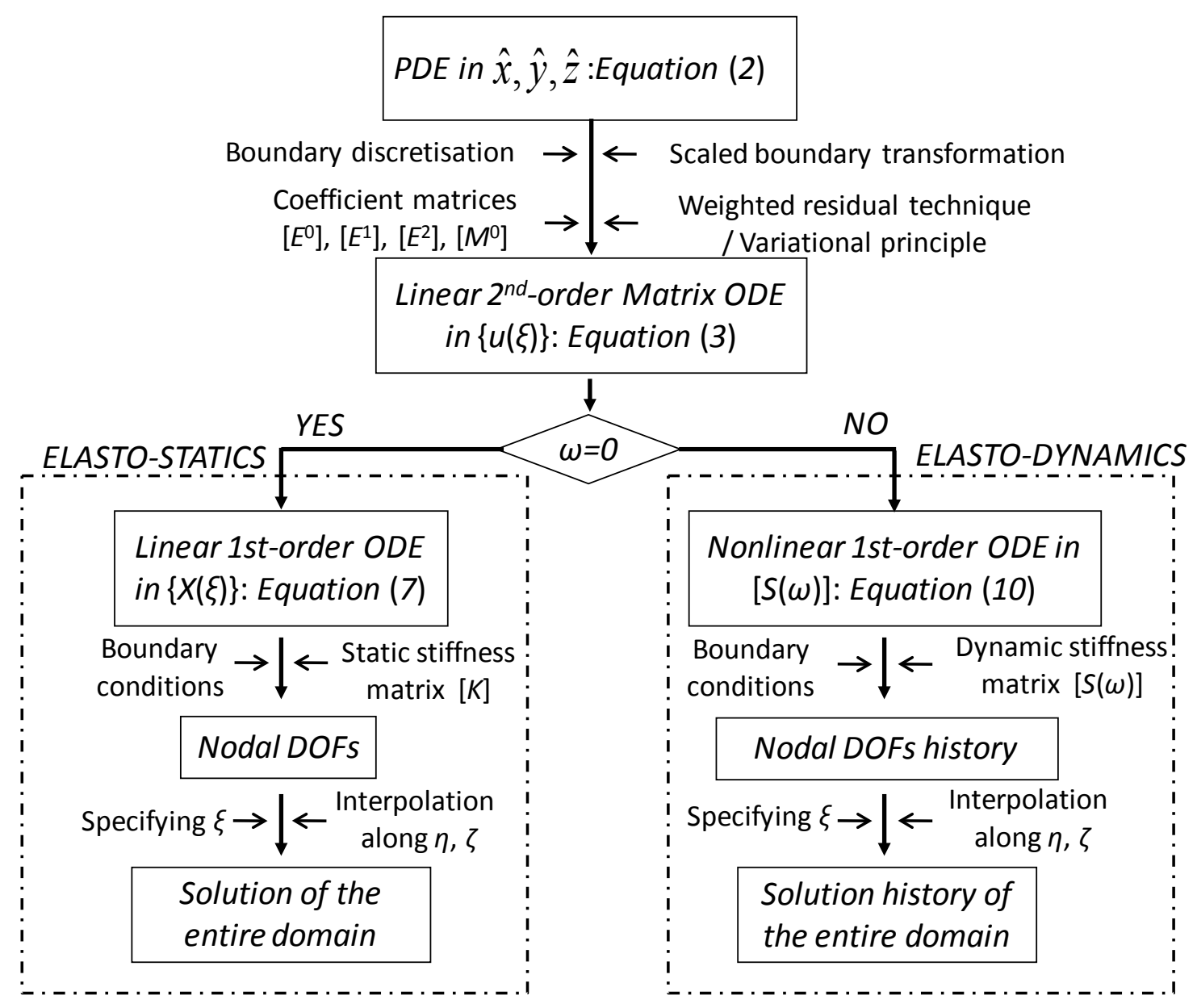

Figure 2. Solution procedures of SBFEM

\section{Matrix decomposition}

3.1 Eigenvalue decomposition and inherent numerical issues

The main techniques in solving the matrix-form scaled boundary finite element equation for both elasto-static and elasto-dynamic problems have been summarised in Section 2. A Hamiltonian matrix $[Z]$ is formulated using the coefficient matrices $\left[E^{0}\right],\left[E^{1}\right]$ and $\left[E^{2}\right]$ of the scaled boundary finite element equation (3), whereby the nodal displacement function $\{u(\xi)\}$ is the basic unknown function. A new intermediate variable $\{X(\xi)\}$ is introduced, which reduces the second-order ODE (3) to a first-order differential equation (7). By hypothesising the displacement field in the form of the power series of the radial coordinate $\xi$, the solution of equation (7) can be formulated as:

$$
\{X(\xi)\}=c_{1} \xi^{-\lambda_{1}}\left\{\phi_{1}\right\}+c_{2} \xi^{-\lambda_{2}}\left\{\phi_{2}\right\}+\cdots+c_{n} \xi^{-\lambda_{n}}\left\{\phi_{n}\right\}
$$


with $n$ denoting the dimension of the Hamiltonian matrix [Z]. Substituting equation (11) into equation (7) leads to the eigenproblem:

$$
[Z]\left\{\phi_{i}\right\}=\lambda_{i}\left\{\phi_{i}\right\} \text { for } i=1,2, \ldots, n
$$

where $\lambda_{\mathrm{i}}$ is the eigenvalue of $[Z]$ and $\left\{\phi_{i}\right\}$ is the corresponding eigenvector. Equation (11) can be reformulated in a matrix form as:

$$
\{X(\xi)\}=\left[\begin{array}{ll}
{\left[\Phi_{11}\right]} & {\left[\Phi_{12}\right]} \\
{\left[\Phi_{21}\right]} & {\left[\Phi_{22}\right]}
\end{array}\right]\left[\begin{array}{ll}
{\left[\xi^{\Lambda_{1}}\right]} & \\
& {\left[\xi^{\Lambda_{2}}\right]}
\end{array}\right]\left\{\begin{array}{l}
\left\{C_{1}\right\} \\
\left\{C_{2}\right\}
\end{array}\right\}
$$

In equation (13), $\Lambda_{1}$ and $\Lambda_{2}$ are two diagonal matrices with $\lambda_{i}$ being their entries. Note, if $\lambda$ is the eigenvalue of $[Z]$, then $-\lambda, \bar{\lambda}$ (conjugate complex number) and $-\bar{\lambda}$ are eigenvalues of $[Z]$. The eigenvalues $\lambda_{\mathrm{i}}$ of matrix $[Z]$ can be arranged in such a way that all the eigenvalues in $\Lambda_{1}$ have positive real parts, and all the eigenvalues in $\Lambda_{2}$ have negative real parts. According to equation (13) and equation $(6),\{u(\xi)\}$ and $\{q(\xi)\}$ can be expressed as:

$$
\begin{aligned}
& \{u(\xi)\}=\xi^{-0.5}\left(\left[\Phi_{11}\right]\left[\xi^{\Lambda_{1}}\right]\left\{C_{1}\right\}+\left[\Phi_{12}\right]\left[\xi^{\Lambda_{2}}\right]\left\{C_{2}\right\}\right) \\
& \{q(\xi)\}=\xi^{+0.5}\left(\left[\Phi_{21}\right]\left[\xi^{\Lambda_{1}}\right]\left\{C_{1}\right\}+\left[\Phi_{22}\right]\left[\xi^{\Lambda_{2}}\right]\left\{C_{2}\right\}\right)
\end{aligned}
$$

leaving the integral constants $\left\{C_{1}\right\}$ and $\left\{C_{2}\right\}$ to be determined according to the prescribed boundary conditions.

The displacement amplitude at the scaling centre where $\xi=0$ for a bounded domain should be finite. Since the real parts of $\lambda_{\mathrm{i}}$ in $\Lambda_{2}$ are negative, equations (14) and (15) are reduced to:

$$
\{u(\xi)\}=\xi^{-0.5}\left[\Phi_{11}\right]\left[\xi^{\Lambda_{1}}\right]\left\{C_{1}\right\}
$$

and

$$
\{q(\xi)\}=\xi^{+0.5}\left[\Phi_{21}\right]\left[\xi^{\Lambda_{1}}\right]\left\{C_{1}\right\}
$$

Eliminating the constant vector $\left\{C_{1}\right\}$ from equations (16) and (17), and noticing that $\{R\}$ $=[K]\{u\}$ and $\{R\}=\{q(\xi=1)\}$ on the boundary, the following expression yields:

$$
[K]=\left[\Phi_{21}\right]\left[\Phi_{11}\right]^{-1}
$$

Consequently, the nodal displacement vector $\{u\}$ and the constant vector $\left\{C_{1}\right\}$ can be 
calculated from equation (5) and equation (16), respectively.

After $\left\{C_{1}\right\}$ is determined, the nodal displacement function $\{u(\xi)\}$ along the line defined by connecting the scaling centre and the corresponding node on the boundary is analytically obtained from equation (16). For unbounded domains, the displacement amplitude at $\xi=\infty$ must remain finite and $\{R\}=-\{q(\xi=1)\}$ applies.

In real cases, however, the power series formulation may not provide a complete general solution, since logarithmic terms exist in problems involving particular geometric configurations, material composition and boundary conditions $[3,8,15,16]$. In this case, multiple eigenvalues or near-multiple eigenvalues of the Hamiltonian matrix $[Z]$ might be present, corresponding to parallel eigenvectors and indicating the existence of logarithmic terms in the solution. Consequently, matrices $\left[\Phi_{11}\right]$ and $\left[\Phi_{21}\right]$ in equation (18) (or $\left[\Phi_{12}\right]$ and $\left[\Phi_{22}\right]$ for the case of an unbounded domain) formulated by parallel eigenvectors are rank-deficient and irreversible, which results in inaccurate solutions or even failure of the eigenvalue decomposition when solving the scaled boundary finite element equation.

\subsection{Real Schur decomposition}

Deeks and Wolf [5,7] investigated a two-dimensional unbounded domain problem governed by the Laplace equation using SBFEM, in which the displacement amplitude is infinite in the near field. This infinite term is represented by an additional logarithmic mode, associated with the rigid body translation, to the power series formulation of the solution. Song [17] proposed a matrix-function solution in combination with the real Schur decomposition to address this multiple - eigenvalue issue. Terms in the series solution are not restricted to power function form. Unlike the work presented in Deeks and Wolf [5, 7], Song's [17] matrix function method does not require prior knowledge of the presence of logarithmic terms, and copes well with the power functions, logarithmic functions and their transitions in the solution. Li et al. (2010a) further discussed the outperformance of the real Schur decomposition over the conventional eigenvalue decomposition technique.

The real Schur decomposition of the Hamiltonian matrix $[Z]$ can be expressed as: 


$$
[Z]=[V][S][V]^{T}
$$

where $[V]$ is an orthogonal matrix and $[S]$ is a block upper triangular matrix with 1-by-1 and 2-by-2 blocks on the diagonal. The eigenvalues are revealed by the diagonal elements and blocks of $[S]$. The columns of $[V]$ constitute a basis offering superior numerical properties to a set of eigenvectors $\left\{\phi_{i}\right\}$ in equation (12) [14]. [S] and [V] are partitioned into submatrices of equal size as:

$$
[S]=\left[\begin{array}{cc}
{\left[S_{n}\right]} & * \\
0 & {\left[S_{p}\right]}
\end{array}\right] \text {, and }[V]=\left[\begin{array}{cc}
{\left[V_{u 1}\right]} & {\left[V_{u 2}\right]} \\
{\left[V_{q 1}\right]} & {\left[V_{q 2}\right]}
\end{array}\right],
$$

with $*$ representing a real matrix. The diagonal elements of matrix $\left[S_{n}\right]$ are negative and those of matrix $\left[S_{p}\right]$ are positive. Block-diagonalising $[S]$ using an upper-triangular matrix and using equation (19) lead to:

$$
[\Psi]^{-1}[Z][\Psi]=\left[\begin{array}{cc}
{\left[S_{n}\right]} & 0 \\
0 & {\left[S_{p}\right]}
\end{array}\right]
$$

Similar to the formulation in equation (13), the general solution of equation (7) using the real Schur decomposition is expressed as:

$$
\{X(\xi)\}=\left[\begin{array}{ll}
{\left[\Psi_{u 1}\right]} & {\left[\Psi_{u 2}\right]} \\
{\left[\Psi_{q 1}\right]} & {\left[\Psi_{q 2}\right]}
\end{array}\right]\left[\begin{array}{ll}
\xi^{-\left[S_{n}\right]} & \\
& \xi^{-\left[S_{p}\right]}
\end{array}\right]\left\{\begin{array}{l}
\left\{C_{1}\right\} \\
\left\{C_{2}\right\}
\end{array}\right\}
$$

Accordingly, $\{u(\xi)\}$ and $\{q(\xi)\}$ can be expressed as:

$$
\begin{array}{r}
\{u(\xi)\}=\xi^{-0.5}\left(\left[\Psi_{u 1}\right] \xi^{-\left[S_{n}\right]}\left\{C_{1}\right\}+\left[\Psi_{u 2}\right] \xi^{-\left[S_{p}\right]}\left\{C_{2}\right\}\right) \\
\{q(\xi)\}=\xi^{+0.5}\left(\left[\Psi_{q 1}\right] \xi^{-\left[S_{n}\right]}\left\{C_{1}\right\}+\left[\Psi_{q 2}\right] \xi^{-\left[S_{p}\right]}\left\{C_{2}\right\}\right)
\end{array}
$$

The following solution procedure is the same as described for the eigenvalue decomposition in Section 3.1. By performing the real Schur decomposition, the inverse of a possibly close-to-singular matrix $\left[\Phi_{11}\right]$ (or $\left[\Phi_{12}\right]$ ) can be avoided by inverting only an upper triangular matrix $\left[\Psi_{u 1}\right]$ (or $\left[\Psi_{u 2}\right]$ ). In addition, real Schur decomposition is more stable and suffers less from numerical difficulties than the eigenvalue decomposition. A case study is provided in the next subsection to demonstrate the efficiency of the real Schur decomposition. 


\subsection{Numerical example}

A cylindrical pile with a radius of $a=1 \mathrm{~m}$ and a height of $h=10 \mathrm{~m}$ subject to uniformly distributed pressure $p=3 \times 10^{8} \mathrm{~Pa}$ is shown in Figure 3. The bottom of the cylinder pile is fixed. The pile is assumed to exhibit elastic behaviour, with Young's modulus $E$ and Poisson's ratio $v$ being $2.8 \times 10^{10} \mathrm{~Pa}$ and 0.25 , respectively. The scaling centre is chosen at the bottom centre of the pile. The circumferential boundary, as well as the top surface of the cylinder is discretised with quadratic eight-node quadrilateral isoparametric elements. A representative scaled boundary element is shown in Figure 4, accompanied by corresponding shape function expressions. An example of the discretisation scheme is illustrated in Figure 5 (a).

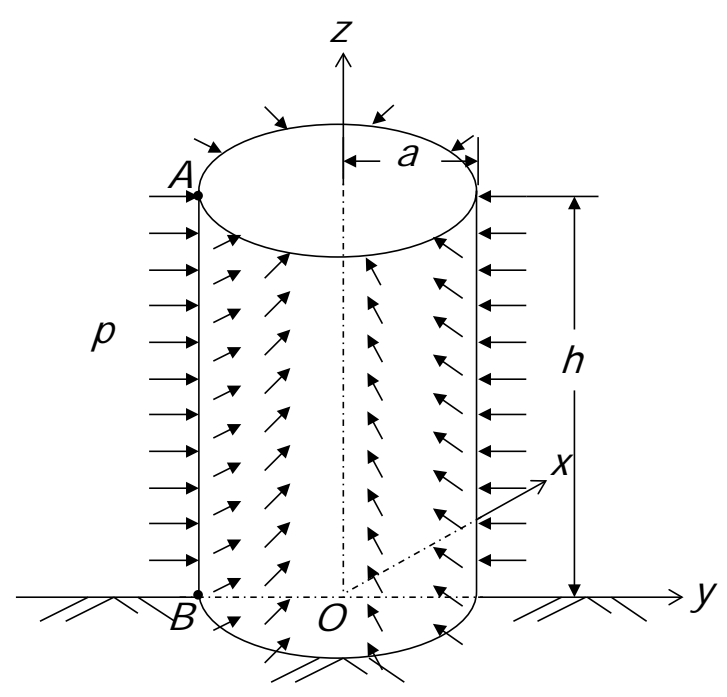

Figure 3. A cylindrical pile subjected to a uniformly distributed pressure

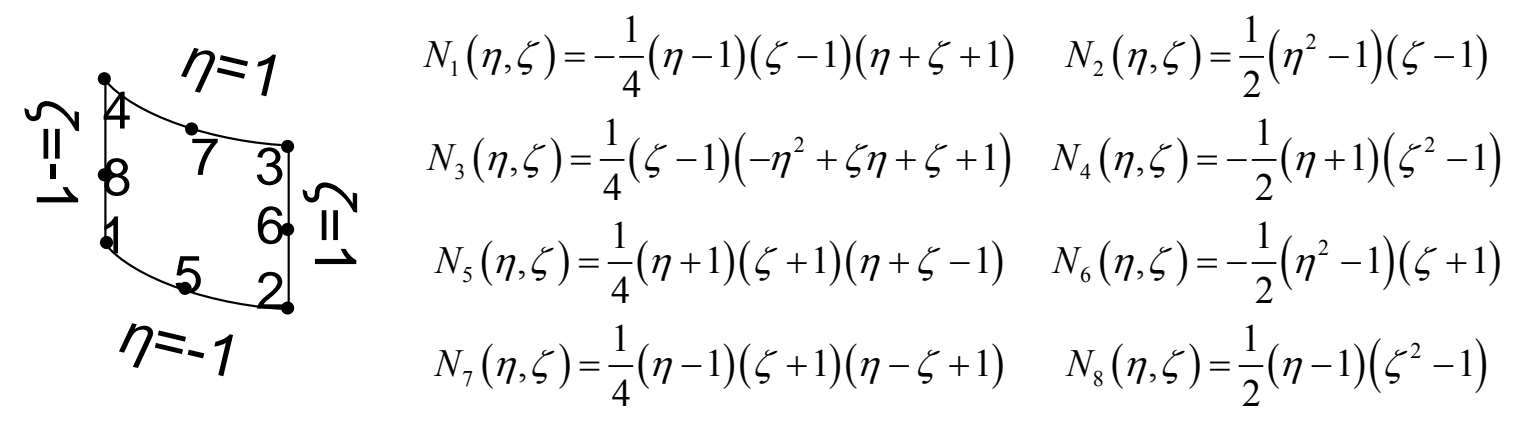

Figure 4. A typical scaled boundary element and the shape functions 


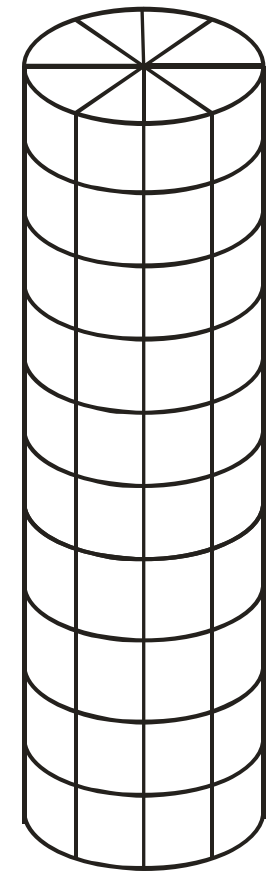

(a)



(b)

Figure 5. Discretisation illustration of the pile foundation for (a) SBFEM model and (b) FEM model

The real Schur decomposition is employed in the calculation. The convergence test shows that 8 elements are needed around the pile circumference, 1 element along the radius and 16 elements along the height of the pile. The displacement of point $A$ (see Figure 3 ) on the edge of the pile head converges to $8.0357 \mathrm{~mm}$ in the $x$ direction, and that in the $z$ direction is $52.344 \mathrm{~mm}$. It should be mentioned that in this example, a nondimensionalised SBFEM model is used to exclude the possibility of numerical inaccuracy caused by unfavourable matrix properties, such as ill-conditioning. The SBFEM nondimensionalisation will be detailed in Section 4.

An equivalent FEM analysis is carried out for comparison purposes. Three dimensional 20-node hexahedral solid elements are used in the FEM model, as shown in Figure 5 (b). A convergence test shows that 28 elements are required around the circumference, 5 elements along the radius and 50 elements for the height. The displacement of point $A$ in the $x$ direction converges to $8.0357 \mathrm{~mm}$, and that in the $z$ direction reaches $52.345 \mathrm{~mm}$. 
The displacement profiles of line $A B$ (see Figure 3) from both SBFEM and FEM models are plotted in Figure 6, in which lines are used to represent FEM results, and markers for SBFEM results. The comparison shows excellent performance of the real Schur decomposition in the SBFEM solution process.

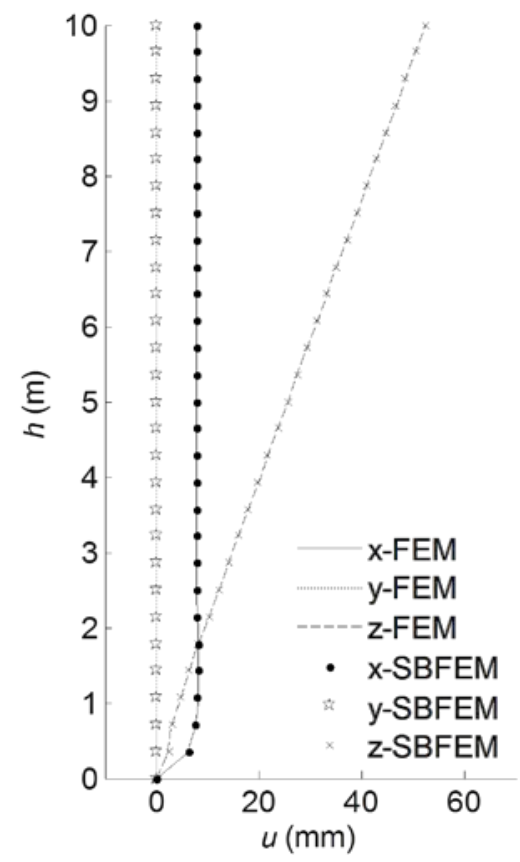

Figure 6. Displacement comparison between SBFEM and FEM models

In order to demonstrate the superiority of the real Schur decomposition over the eigenvalue decomposition, the radial and vertical displacements of point $A$, calculated using the two matrix decomposition algorithms, are compared in Figure 7. The tick labels on the horizontal axis represent different discretisation schemes. For example, $6 \times 10 \times 1$ signifies that the numbers of elements in the circumferential, vertical and radial directions are 6,10 and 1, respectively. It is found that by using the real Schur decomposition, no prior knowledge of the potential multiple eigenvalues is required and no complex number operation is performed, as is necessary in the case of the eigenvalue decomposition. The inversion of rank-deficient matrices can be efficiently avoided. The real Schur decomposition tends to give more stable and reliable results compared to the eigenvalue decomposition, as shown in Figure 7. 


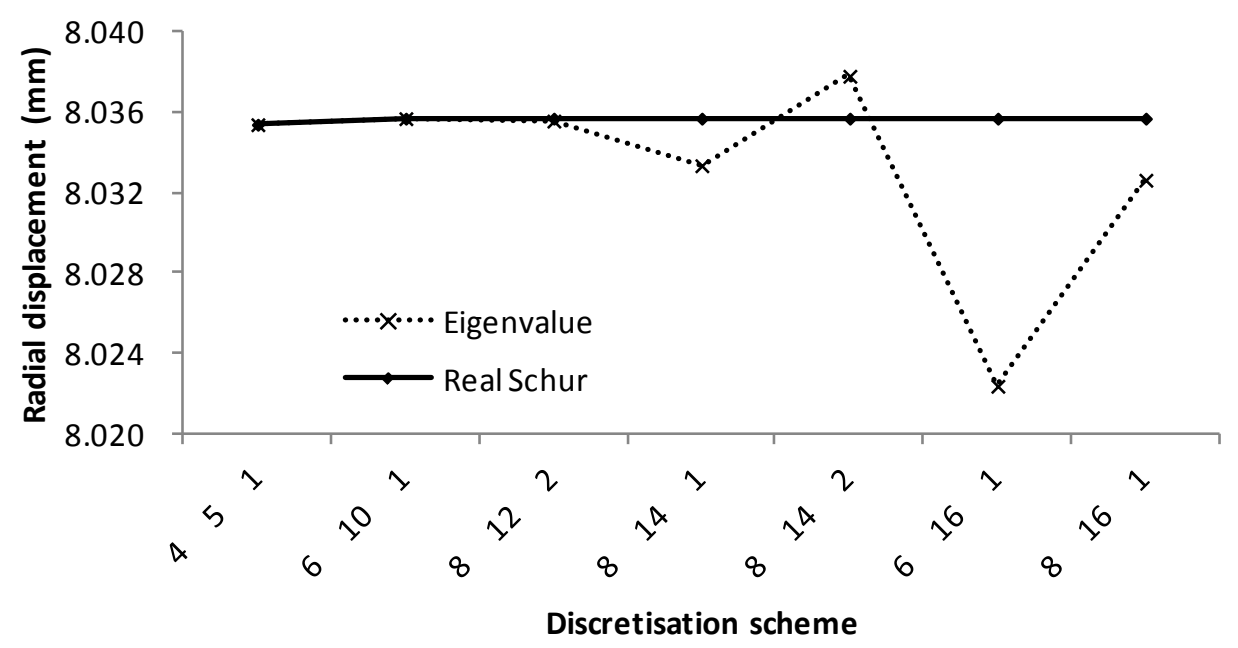

(a)

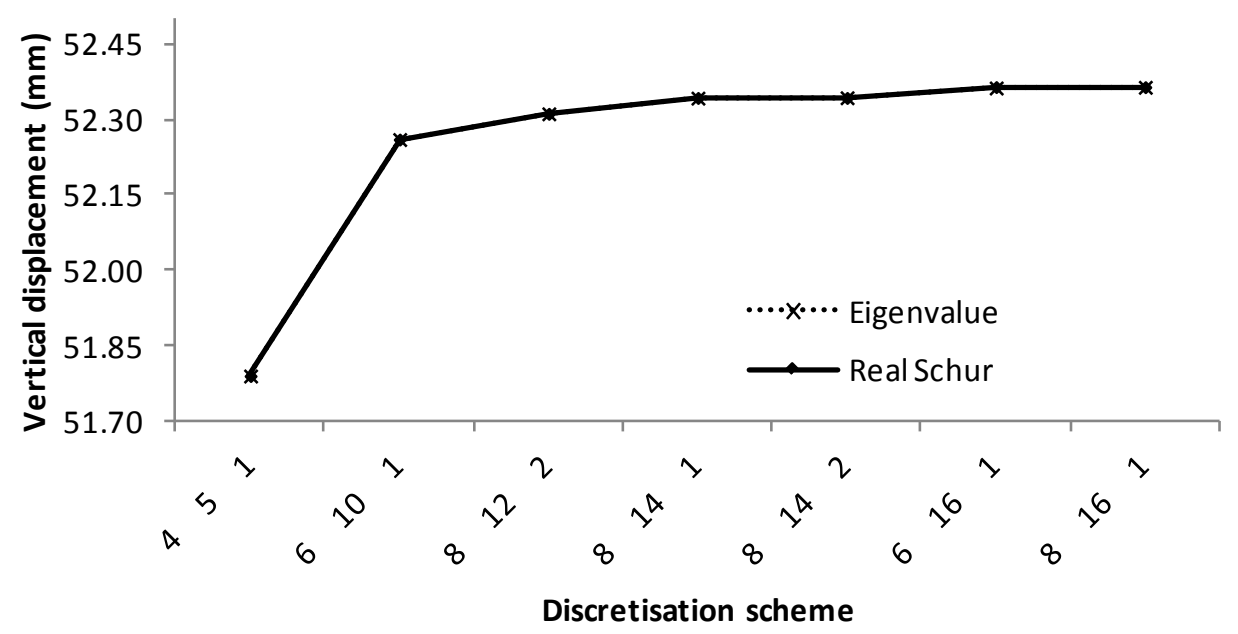

(b)

Figure 7. Comparison between the eigenvalue decomposition and the real Schur decomposition methods for: (a) radial displacement and (b) vertical displacement (Note: the vertical displacements from the two methods overlap in the plot)

\section{SBFEM non-dimensionalisation}

4.1 Numerical issues associated with matrix properties

The case of a cylindrical pile subject to uniformly distributed pressure, as illustrated in 
Figure 3, is also utilised in this section to investigate the numerical credibility of the SBFEM calculation in relation to matrix properties. The displacement components of point $A$ in the $x, y$ and $z$ directions are to be examined.

In the SBFEM model, the scaling centre $O$ is chosen at the geometric centre of the pile at $(0,0,5)$, and the entire surface is discretised into 184 eight-node quadratic quadrilateral elements. The solution procedure employing the real Schur decomposition presented in Section 3.2 is followed. Using the raw parameters of the cylindrical pile presented in Section 3.3, the displacement components in the $x, y$ and $z$ directions of point $A$ are calculated as $4.431 \times 10^{3} \mathrm{~mm}, 1.421 \times 10^{4} \mathrm{~mm}$ and $7.178 \times 10^{4} \mathrm{~mm}$, respectively. Apparently, these results differ considerably from the solutions depicted in Figure 7, which are $8.036 \mathrm{~mm}, 0$ and $52.382 \mathrm{~mm}$.

A close examination of the Hamiltonian matrix $[Z]$ reveals that its condition number $\kappa$ equals $2 \times 10^{24}$. This implies that the Hamiltonian matrix is ill-conditioned, and any succeeding manipulations either directly or indirectly related to this matrix may fail upon any rounding error fluctuation. The exactness of equation (19) is checked by examining the norm of a residual matrix $\left[\operatorname{Res}_{1}\right]$ :

$$
\left[\operatorname{Res}_{1}\right]=[Z]-[V][S][V]^{T}
$$

Theoretically, a norm $\left[\operatorname{Res}_{1}\right]=0$ calculated from equation (23) is expected. However, a norm of 0.1345 is observed, which is far beyond the acceptable accuracy tolerance with an order of $10^{-7}[9]$.

Another examination can be associated with the static stiffness matrix $[K]$. It is understood that the static stiffness matrix $[K]$ obtained from equation (18) should satisfy equation (10), replacing $[S(\omega)]$ with $[K]$ and $\omega$ with 0 . Therefore, another residual matrix $\left[\operatorname{Res}_{2}\right]$ is defined in equation (24), the norm of which is found to be $9 \times 10^{21}$.

$$
\left[\operatorname{Res}_{2}\right]=\left([K]-\left[E^{1}\right]\right)\left[E^{0}\right]^{-1}\left([K]-\left[E^{1}\right]^{T}\right)-\left[E^{2}\right]+[K]
$$

By examining the Hamiltonian matrix, it is found that the maximum magnitude of its entries is of $10^{10}$, resulting from the input parameter, i.e. the Young's modulus which holds a magnitude of $10^{8}$ in the present case. The minimum magnitude, however, is 0 . 
This significant difference in magnitudes of the matrix entries leads to the illconditioning of the matrix.

An elastic wave propagation problem in unbounded domain serves as another example illustrating the detrimental effects of large magnitudes of input parameters to SBFEM calculations. The case of a quarter of a square prism footing embedded in a semi-infinite half space presented in [19] is used herein to illustrate the problem. The geometry of the footing is reproduced in Figure 8 (a) with $b=1 \mathrm{~m}$ and $e=2 / 3 \mathrm{~m}$. The material properties of the half-space are assigned as: the shear modulus $G=1 \times 10^{10} \mathrm{~Pa}$; Poisson's ratio $v$ is $1 / 3$, and the mass density $\rho$ is $2500 \mathrm{~kg} / \mathrm{m}^{3}$.

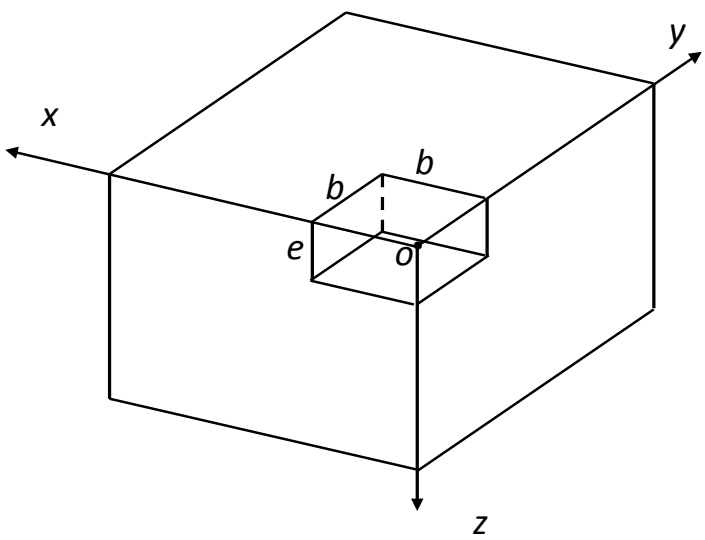

(a)

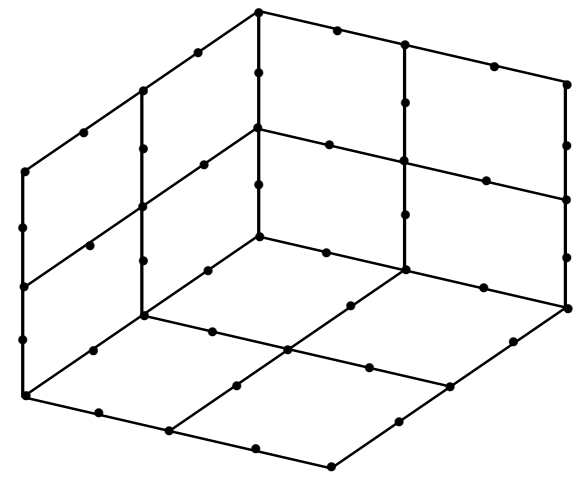

(b)

Figure 8. A quarter of a square prism footing embedded in a semi-infinite space: (a) the geometric plot and (b) scaled boundary discretisation of the prism-medium interface

A SBFEM model is established with the scaling centre located at point $O$ in Figure 8 (a). The interface between the footing and the unbounded domain is discretised into 12 eightnode quadratic quadrilateral elements, resulting in a total of 49 nodes, as shown in Figure 8 (b). The continued-fraction technique is used to formulate the dynamic stiffness matrix $\left[S^{\infty}(\omega)\right]$ in equation (9). Details of the continued-fraction formulation of $\left[S^{\infty}(\omega)\right]$ for unbounded domain can be found in [1], with key equations presented below for ease of discussions. The dynamic stiffness matrix $\left[S^{\infty}(\omega)\right]$ is decomposed as: 


$$
\left[S^{\infty}(\omega)\right]=i \omega\left[C_{\infty}\right]+\left[K_{\infty}\right]-\left[Y^{(1)}(\omega)\right]^{-1}
$$

where $\left[C_{\infty}\right]$ and $\left[K_{\infty}\right]$ are the constant dashpot matrix and the stiffness matrix, respectively. $\left[Y^{(i)}(\omega)\right]$ is the residual of the two-term expansion of $\left[S^{\infty}(\omega)\right]$ at high frequency, and is expressed in a recursive form as:

$$
\left[Y^{(i)}(\omega)\right]=i \omega\left[Y_{1}^{(i)}\right]+\left[Y_{0}^{(i)}\right]-\left[Y^{(i+1)}(\omega)\right]^{-1} \quad(i=1,2,3 \cdots)
$$

with $\left[\mathbf{Y}_{1}^{(i)}\right]$ and $\left[\mathbf{Y}_{0}^{(i)}\right]$ being the auxiliary matrices, and the superscript $i$ denoting the order of the continued-fraction formulation. Substituting equation (25) into equation (9) yields:

$$
\begin{aligned}
& \left\{\begin{array}{l}
\{R(\omega)\}=\left(i \omega\left[C_{\infty}\right]+\left[K_{\infty}\right]\right)\{u(\omega)\}-\left\{u^{(1)}(\omega)\right\} \\
\{u(\omega)\}=\left[Y^{(1)}(\omega)\right]\left\{u^{(1)}(\omega)\right\}
\end{array}\right. \\
& \begin{cases}\left\{u^{(i-1)}(\omega)\right\}=\left(i \omega\left[Y_{1}^{(i)}\right]+\left[Y_{0}^{(i)}\right]\right)\left\{u^{(i)}(\omega)\right\}-\left\{u^{(i+1)}(\omega)\right\} & i>1 \\
\left\{u^{(i)}(\omega)\right\}=\left[Y^{(i+1)}(\omega)\right]\left\{u^{(i+1)}(\omega)\right\} & \end{cases}
\end{aligned}
$$

Equation (27) can be reformulated in a matrix form as:

$$
([A]+i \omega[B])\{\hat{u}(\omega)\}=\{\hat{F}(\omega)\}
$$

In equation (28), $\{\hat{\boldsymbol{u}}(\omega)\}$ and $\{\hat{\boldsymbol{F}}(\omega)\}$ represent the displacement and external force vectors, respectively. $[A]$ and $[B]$ are coefficient matrices formulated using $\left[\mathbf{Y}_{1}^{(i)}\right]$ and $\left[\boldsymbol{Y}_{0}^{(i)}\right](i=1,2,3 \cdots)$, as shown in equation (29):

$$
\begin{gathered}
{[\boldsymbol{A}]=\left[\begin{array}{cccccc}
{\left[\boldsymbol{K}_{\infty}\right]} & -[\boldsymbol{I}] & & \ldots & & \\
-[\boldsymbol{I}] & {\left[\boldsymbol{Y}_{0}^{(1)}\right]} & -[\boldsymbol{I}] & \ldots & & \\
& -[\boldsymbol{I}] & {\left[\boldsymbol{Y}_{0}^{(2)}\right]} & \ldots & & \\
\vdots & \vdots & \vdots & \ddots & -[\boldsymbol{I}] & \\
& & & -[\boldsymbol{I}] & {\left[\boldsymbol{Y}_{0}^{\left(M_{c f}-1\right)}\right]} & -[\boldsymbol{I}] \\
& & & & -[\boldsymbol{I}] & {\left[\boldsymbol{Y}_{0}^{\left(M_{c f}\right)}\right]}
\end{array}\right]} \\
{[\boldsymbol{B}]=\operatorname{diag}\left(\left[\boldsymbol{C}_{\infty}\right],\left[\boldsymbol{Y}_{1}^{(1)}\right],\left[\boldsymbol{Y}_{1}^{(2)}\right], \cdots,\left[\boldsymbol{Y}_{1}^{\left(M_{c f}-1\right)}\right],\left[\boldsymbol{Y}_{1}^{\left(M_{c f}\right)}\right]\right)}
\end{gathered}
$$


Matrices $\left[\boldsymbol{Y}_{1}^{(i)}\right]$ and $\left[\boldsymbol{Y}_{0}^{(i)}\right](i=1,2,3 \cdots)$ are obtained through a series of calculations of matrices formulated using parameters such as the material density and Young's modulus (details can be referred to in [1]). These calculations include solving Sylvester equations and matrix inversions. Hence, the properties of matrices involved are of significant importance to the stability and accuracy of overall SBFEM calculations. Taking matrix $[A]$ in equation (29) as an example, representing alternatively the stiffness and the flexibility of the study domain, the magnitude of matrix entries in $\left[K_{\infty}\right]$ is reciprocal to that in $\left[\boldsymbol{Y}_{0}^{(1)}\right]$ as shown in Table 3. Note that for ease of discussion, instead of examining any particular element in the matrix, an algebraic sum of elements corresponding to $x, y$ and $z$ directions is performed, resulting in scalar quantities. Especially, when the magnitude of input parameters formulating these matrices is large such as the Young's/shear modulus which normally holds a magnitude of up to 10 , the magnitude of matrix entries in $\left[K_{\infty}\right]$ and $\left[\boldsymbol{Y}_{0}^{(1)}\right]$ varies significantly from each other (this is analogous between $\left[\boldsymbol{Y}_{0}^{(1)}\right]$ and $\left[\boldsymbol{Y}_{0}^{(2)}\right], \ldots,\left[\boldsymbol{Y}_{0}^{\left(M_{c f}-1\right)}\right]$ and $\left.\left[\boldsymbol{Y}_{0}^{\left(M_{c f}\right)}\right]\right)$. In the meantime, the degree of the difference amplifies as the order of the continued-fraction formulation increases. Subsequently, upon formulating the coefficient matrix $[A]$ (analogously matrix $[B]$ ) when solving equation (28), the difference in element entries in $\left[K_{\infty}\right],\left[\boldsymbol{Y}_{0}^{(1)}\right],\left[\mathbf{Y}_{0}^{(2)}\right], \ldots$, and $\left[\boldsymbol{Y}_{0}^{\left(M_{c f}\right)}\right]$ results in the ill-conditioning of matrix $[A]$ (and $[B]$ ), which accordingly leads to the failure of the SBFEM solution.

From the above discussions, it is necessary that input parameters are processed prior to calculations to improve the quality of matrices, for the benefit of subsequent computations. It is motivated to introduce the non-dimensionalisation scheme into the SBFEM calculation, as it allows all quantities to have relatively similar orders of magnitudes. The detailed procedure of non-dimensionalisation and its incorporation into the SBFEM formulation are presented in the next subsection.

4.2 SBFEM non-dimensionalisation procedure 
Wolf and Song [25] presented a dimensional analysis identifying independent variables to which the dynamic stiffness matrix is related. The non-dimensionalisation scheme proposed in this study follows their idea. The dimensionless length $r^{*}$, Young's modulus $E^{*}$ and the mass density $\rho^{*}$ are calculated using corresponding reference variables as $r^{*}=$ $r / r_{\mathrm{r}}, E^{*}=E / E_{\mathrm{r}}$ and $\rho^{*}=\rho / \rho_{\mathrm{r}}$, respectively. The dimensions of the dynamic stiffness matrix $[S(\omega)]$ and the independent variable frequency $\omega$ are $L^{s-3} M T^{2}$ and $T^{1}$, with $s$ representing the spatial dimension of the study domain $(s=2$ for two-dimensional problems and 3 for three-dimensional problems). An equation expressing the dimensions of $[S]^{n_{1}} r_{r}^{n_{2}} E_{r}^{n_{3}} \rho_{r}^{n_{4}} \omega^{n_{5}}$ being $L^{(s-3) n_{1}+n_{2}-n_{3}-3 n_{4}} M^{n_{1}+n_{3}+n_{4}} T^{-2 n_{1}-2 n_{3}-n_{5}}$ is used to formulate the dimensionless $\left[S^{*}(\omega)\right]$ and $\omega^{*}$. It is worth mentioning that this equation is formulated using the reference variables, rather than the corresponding material parameters, as is the case in Wolf and Song [25]. This allows more flexibility in the non-dimensionalisation process, and yields:

$$
\begin{aligned}
& (s-3) n_{1}+n_{2}-n_{3}-3 n_{4}=0 \\
& n_{1}+n_{3}+n_{4}=0 \\
& -2 n_{1}-2 n_{3}-n_{5}=0
\end{aligned}
$$

and are used to determine the five parameters $n_{\mathrm{i}}(i=1,2,3,4$ and 5). It is noticed that two of them are arbitrarily chosen. Given $n_{1}=1$ and $n_{5}=0$ yields the dimensionless dynamic stiffness matrix $\left[S^{*}(\omega)\right]$ :

$$
\left[S^{*}(\omega)\right]=r_{r}^{2-s} E_{r}^{-1}[S(\omega)]
$$

or if $n_{1}=0$ and $n_{5}=1$ :

$$
\omega^{*}=\frac{\omega r_{r}}{\sqrt{E_{r} / \rho_{r}}}
$$

Analogically, the following expressions hold for the static stiffness matrix $[K]$, the mass matrix $[M]$, and the damping matrix $[C]$ :

$$
\left[K^{*}\right]=E_{r}^{-1} r_{r}^{-1}[K]\left[M^{*}\right]=\rho_{r}^{-1} r_{r}^{-3}[M]\left[C^{*}\right]=\frac{\sqrt{E_{r} / \rho_{r}}}{E_{r} r_{r}^{2}}[C]
$$


The coefficient matrices $\left[E^{0}\right],\left[E^{1}\right],\left[E^{2}\right]$ and $\left[M^{0}\right]$ are non-dimensionalised accordingly as:

$$
\begin{array}{ll}
{\left[E^{0 *}\right]=E_{r}^{-1} r_{r}^{2-s}\left[E^{0}\right]} & {\left[E^{1} *\right]=E_{r}^{-1} r_{r}^{2-s}\left[E^{1}\right]} \\
{\left[E^{2} *\right]=E_{r}^{-1} r_{r}^{2-s}\left[E^{2}\right]} & {\left[M^{0} *\right]=\rho_{r}^{-1} r_{r}^{-s}\left[M^{0}\right]}
\end{array}
$$

Another independent variable, the time $t^{*}$, needs to be reformulated in the time-domain analysis as:

$$
t^{*}=\frac{\sqrt{E_{r} / \rho_{r}}}{r_{r}} t
$$

With all the above expressions, equation (3) retains exactly its original form. For ease of presentation, all asterisks are removed from the mathematical expressions thereafter unless specified otherwise.

Corresponding to the continued-fraction formulation of the dynamic stiffness matrix $\left[S^{\infty}(\omega)\right]$ in equation $(25)$, the non-dimensionalised form of relevant matrices are derived as:

$$
\begin{aligned}
& {\left[C_{\infty}^{*}\right]=\frac{\sqrt{E_{r} / \rho_{r}}}{E_{r} r_{r}^{s-1}}\left[C_{\infty}\right]} \\
& {\left[K_{\infty}^{*}\right]=E_{r}^{-1} r_{r}^{2-s}\left[K_{\infty}\right]} \\
& {\left[Y_{0}^{(i)^{*}}\right]=\left(E_{r} r_{r}^{s-2}\right)^{-N_{i}}\left[Y_{0}^{(i)}\right]} \\
& {\left[Y_{1}^{(i)^{*}}\right]=\left(E_{r}^{0.5} \rho_{r}^{0.5} r_{r}^{s-1}\right)^{-N_{i}}\left[Y_{1}^{(i)}\right] \quad\left(N_{i}=(-1)^{i}\right)}
\end{aligned}
$$

Accordingly, equations (27) - (29) are reformulated and maintain exactly the same format, only replacing the dimensional quantities with the corresponding non-dimensionalised counterparts:

$$
\begin{aligned}
& \left\{R^{*}\right\}=E_{r}^{-1} r_{r}^{1-s}\{R\} \\
& \left\{u^{*}\right\}=r_{r}^{-1}\{u\} \\
& \left\{u^{(i)^{*}}\right\}=\left(E_{r} r_{r}^{s-1}\right)^{-N_{i}}\left\{u^{(i)}\right\}
\end{aligned}
$$

In order for the proposed parametric non-dimensionalisation scheme to be incorporated 
into the SBFEM calculation, a group of reference variables need to be specified beforehand so that, upon non-dimensionalisation, all relevant quantities involved in the calculation are of similar magnitude. Precision has to be considered at various intermediate stages, such as the real Schur decomposition of the Hamiltonian matrix $[Z]$ and the general eigenvalue decomposition of $\left[E^{0}\right]$ and $\left[M^{0}\right]$. In addition, the stiffness matrix $[K]$ and $\left[K_{\infty}\right]$, the mass matrix $[M]$, the damping matrix $[C]$ and $\left[C_{\infty}\right]$, and the auxiliary matrices $\left[\boldsymbol{Y}_{1}^{(i)}\right]$ and $\left[\boldsymbol{Y}_{0}^{(i)}\right](i=1,2,3 \cdots)$ associated with the continued fraction formulation are required to satisfy their corresponding algebraic equations.

It should be mentioned that equations (31), (33) - (34), and (36) - (37) suggest how matrices are non-dimensionalised with respect to the reference variables. They are not explicitly formulated in the solution procedure. Calculated results are dimensionless and require subsequent interpretation in order to be applied to engineering practice. For example, a variable with dimension $L$ should be multiplied by the reference length $r_{\mathrm{r}}$ to obtain the corresponding dimensional value. The following examples will detail these procedures.

\subsection{Numerical examples}

\subsubsection{Static analysis}

The cylindrical pile in Section 3.3 is reconsidered with four sets of reference variables shown in Table 1, to demonstrate the performance of SBFEM non-dimensionalisation. Taking into account the magnitude of the input parameters, four values of $E_{\mathrm{r}}$ are selected as $1 \mathrm{~Pa}, 1 \times 10^{3} \mathrm{~Pa}, 1 \times 10^{7} \mathrm{~Pa}$ and $2.8 \times 10^{10} \mathrm{~Pa}$ to non-dimensionalise the Young's modulus $E$ and the external pressure $p$, both of which have dimensions $M L^{-1} \mathrm{~T}^{-2}$. The reference length $r_{\mathrm{r}}$ equals $1 \mathrm{~m}$, as the same SBFEM model is used for the four cases and the dimension of the model is identical to the physical prototype of the pile. The mass density is irrelevant to this example and therefore is not discussed. For each of the four cases, the maximum difference $\Delta M_{\max }$ in the magnitude of the entries of the Hamiltonian matrix and the condition number $\kappa$ of the Hamiltonian matrix, as well as the norms of the two residual matrices $\operatorname{Res}_{1}$ and $\operatorname{Res}_{2}$ are examined. The displacement components $u_{\mathrm{x}}, u_{\mathrm{y}}$ and $u_{\mathrm{z}}$ of point $A$ are transferred into the corresponding dimensional values and are listed in 
Table 1.

Table 1. Numerical performance illustration of SBFEM using a static analysis

\begin{tabular}{ccccc}
\hline Set number & 1 & 2 & 3 & 4 \\
Parameters & 1 & $1 \times 10^{3}$ & $1 \times 10^{7}$ & $2.8 \times 10^{10}$ \\
\hline$E_{\mathrm{r}}(\mathrm{Pa})$ & 1 & 1 & 1 & 1 \\
$r_{\mathrm{r}}(\mathrm{m})$ & $4 \times 10^{10}$ & $4 \times 10^{7}$ & $4 \times 10^{3}$ & 138.74 \\
$\Delta M_{\max }$ & $2 \times 10^{24}$ & $8 \times 10^{16}$ & $8 \times 10^{8}$ & $2 \times 10^{5}$ \\
$\kappa$ & 0.1345 & $1 \times 10^{-4}$ & $3 \times 10^{-8}$ & $7 \times 10^{-11}$ \\
$\operatorname{Res}_{1}$ & $9 \times 10^{21}$ & $1 \times 10^{9}$ & 0.0011 & $9 \times 10^{-12}$ \\
$\operatorname{Res}_{2}$ & $4.431 \times 10^{3}$ & 11.507 & 8.0357 & 8.0357 \\
$u_{\mathrm{x}}(\mathrm{mm})$ & $1.4205 \times 10^{4}$ & 5.744 & $4.625 \times 10^{-8}$ & $1.394 \times 10^{-8}$ \\
$u_{\mathrm{y}}(\mathrm{mm})$ & $7.178 \times 10^{4}$ & 51.534 & 52.358 & 52.358 \\
$u_{\mathrm{z}}(\mathrm{mm})$ & & & & \\
\hline
\end{tabular}

It is found by evaluating the four indices, $\Delta M_{\max }, \kappa, \operatorname{Res}_{1}$ and $\operatorname{Res}_{2}$, as the reference parameter $E_{\mathrm{r}}$ gradually increases, the numerical performance of the SBFEM calculation improves accordingly. The maximum magnitude difference among the element entries of matrix $[Z]$ decreases from a magnitude of $10^{10}$ to $10^{2}$. The condition number of $[Z]$ thus is calculated to decrease from $10^{24}$ to $10^{5}$. Consequently, the norms of the two residual matrices are found to converge to zero when $E_{\mathrm{r}}$ reaches $2.8 \times 10^{10} \mathrm{~Pa}$. The readings of the displacement components also indicate a trustworthy calculation when appropriate reference variables are employed. It is suggested that the reference parameters are defined in such way that all variables involved in the matrix calculation hold similar magnitude regardless of their dimensions. For the present case, a combination of $E_{\mathrm{r}}=$ $2.8 \times 10^{10} \mathrm{~Pa}$ and $r_{\mathrm{r}}=1 \mathrm{~m}$ generates a magnitude of 1 for both the length dimension $L$ and the pressure dimension $M L^{-1} \mathrm{~T}^{-2}$.

\subsubsection{Modal analysis}

The previous case examines how the accuracy of SBFEM results is affected by the 
magnitude of input parameters in static analysis, thus highlighting the necessity of parametric non-dimensionalisation in the SBFEM calculation. The modal analysis presented in this section and the transient analysis in the next section will examine the performance of the dimensionless SBFEM calculation in elasto-dynamics. The $L$-shaped panel presented in Song [18] is re-examined herein with the same geometric configuration, however, the Young's modulus $E$ and the mass density $\rho$ are assigned as $2.8 \times 10^{10} \mathrm{~Pa}$ and $2400 \mathrm{~kg} / \mathrm{m}^{3}$, respectively. Note that the Poisson's ratio remains as $1 / 3$.

A sketch of the $L$-shaped panel is reproduced in Figure 9 (a), illustrating the geometric configuration $(b=1 \mathrm{~m})$ and the boundary conditions: Line $E F$ is fully constrained in both $x$ and $y$ directions; $A B$ is fixed only in the $x$ direction. In the SBFEM model shown in Figure 9 (b), the $L$-shaped panel is divided into three subdomains with the scaling centres located at the geometric centre of each subdomain. Therefore, all the boundaries as well as the two interfaces between the subdomains are discretised. This analysis can also be carried out by treating the $L$-shaped panel as a single domain and locating the scaling centre at point $O$, thus only those lines apart from $O A$ and $O F$ need to be discretised. Three-node quadratic elements are used for the boundary discretisation, which results in 196 degrees of freedom for the problem. The continued fraction technique is employed and an order of 6 is used when formulating the global stiffness and mass matrices. The reference parameters are chosen as $r_{\mathrm{r}}=1 \mathrm{~m}, E_{\mathrm{r}}=2.8 \times 10^{10} \mathrm{~Pa}$ and $\rho_{\mathrm{r}}=2400 \mathrm{~kg} / \mathrm{m}^{3}$.

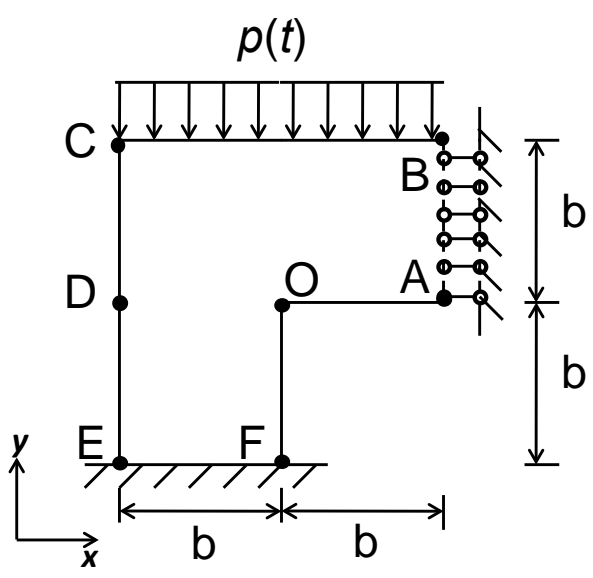

(a)

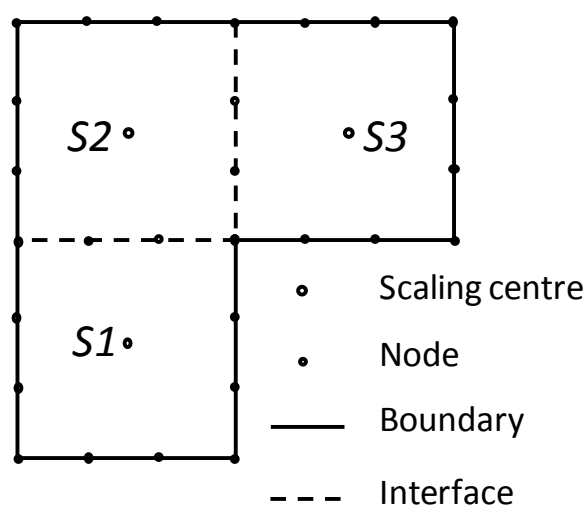

(b)

Figure 9. $L$-shaped panel: (a) geometry of the panel and its boundary conditions (reproduced from [17]) and (b) SBFEM model 
The first 110-order dimensionless natural frequencies calculated from SBFEM are plotted in Figure 10 (a). The dimensional natural frequencies are thus obtained by re-formulating equation (32) as:

$$
\omega=\frac{\sqrt{E_{r} / \rho_{r}}}{r_{r}} \omega^{*}
$$

The result is compared with that of an equivalent FEM modal analysis in Figure 10 (b). The two curves agree extremely well. The same analysis using dimensional parameters in the SBFEM model is also attempted but fails due to the error accumulation during the calculation process, which renders the subsequent results meaningless.

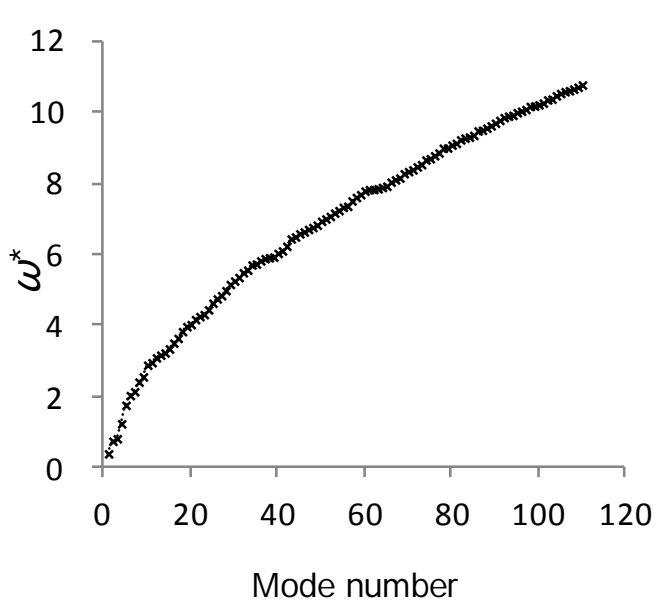

(a)

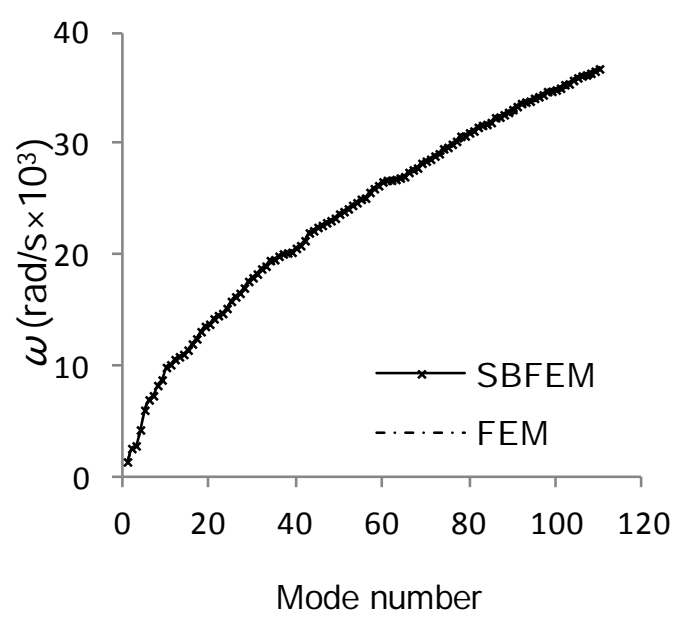

(b)

Figure 10. Natural frequency of the $L$-shaped panel: (a) dimensionless natural frequency from SBFEM model and (b) comparison of dimensional natural frequencies between FEM and SBFEM results

\subsubsection{Transient analysis}

The $L$-shaped panel is also utilised in this section to illustrate the incorporation of the parametric non-dimensionalisation into the transient analysis. The prescribed force condition, similar to that described in Song [18], is specified as: uniformly distributed along line $B C$ (see Figure 9 (a)) with the magnitude varying with respect to time as depicted in Figure 11 (a). In Figure 11 (a), the values of $p_{\text {peak }}, t_{\text {total }}, t_{\text {peak }}, t_{\text {zero }}$ can be referred to in Table 2, in which both dimensional and dimensionless parameters 
employed in this analysis are listed.

Table 2. Parameters of the transient analysis of an $L$-shaped panel

\begin{tabular}{ccccc}
\hline & \multicolumn{2}{c}{ Dimensional } & \multicolumn{2}{c}{ Dimensionless } \\
\hline $\begin{array}{c}\text { Material } \\
\text { parameters }\end{array}$ & $E(\mathrm{~Pa})$ & $2.8 \times 10^{10}$ & $E^{*}$ & 1 \\
& $\rho\left(\mathrm{kg} / \mathrm{m}^{3}\right)$ & 2400 & $\rho^{*}$ & 1 \\
\hline $\begin{array}{c}\text { Temporal variables } \\
t_{\text {total }}(\mathrm{s})\end{array}$ & $t_{\text {peak }}(\mathrm{s})$ & $1.4639 \times 10^{-4}$ & $t^{*}$ peak & 0.5 \\
& $t_{\text {zero }}(\mathrm{s})$ & $2.9277 \times 10^{-4}$ & $t^{*}{ }_{\text {zero }}$ & 1 \\
\hline $\begin{array}{c}\text { Natural circular } \\
\text { frequencies }\end{array}$ & $\omega_{1}(\mathrm{rad} / \mathrm{s})$ & $7.3193 \times 10^{-6}$ & $\Delta t^{*}$ & 0.025 \\
\hline $\begin{array}{c}\text { External pressure } \\
\omega_{2}(\mathrm{rad} / \mathrm{s})\end{array}$ & $p_{\text {peak }}(\mathrm{Pa})$ & 2819.265 & $\omega_{1}{ }^{*}$ & 0.4032 \\
\hline
\end{tabular}

The SBFEM analysis adopts the same discretisation model as that shown in Figure 9 (b), and the reference material properties, $E_{\mathrm{r}}$ and $\rho_{\mathrm{r}}$, are selected as $2.8 \times 10^{10} \mathrm{~Pa}$ and 2400 $\mathrm{kg} / \mathrm{m}^{3}$, respectively. In the calculation, all the temporal variables associated with the time integration, in which the Newmark's integral technique with $\alpha=0.25$ and $\delta=0.5$ [4] being employed, are non-dimensionalised according to equation (35). The Rayleigh material damping effect is taken into consideration with a material damping ratio of 0.05 , assuming the $L$-shaped panel is made of concrete. From the modal analysis presented in Section 4.3.2, $\omega_{1}=1371.682 \mathrm{rad} / \mathrm{s}$ and $\omega_{2}=2819.265 \mathrm{rad} / \mathrm{s}$ corresponding to two orthogonal modal shapes are selected. However, their dimensionless counterparts are used for the formulation of the damping matrix. The magnitude of the external pressure at any time step is non-dimensionalised in accordance with the Young's modulus. 


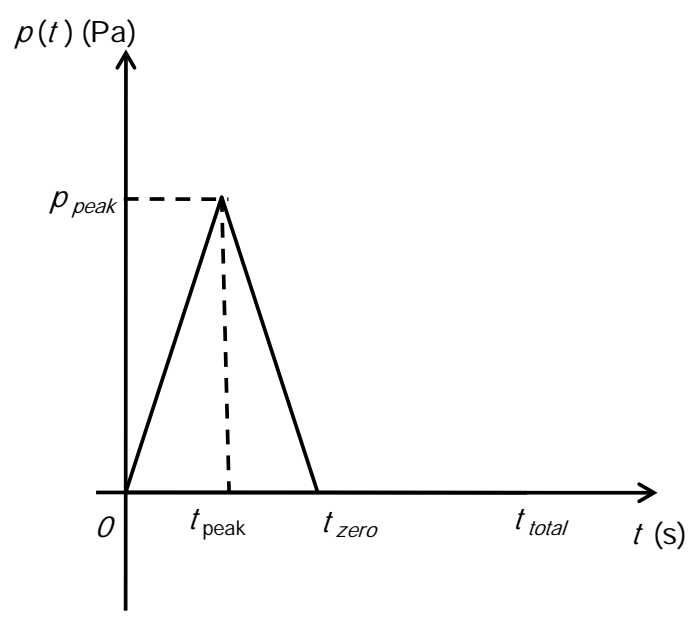

(a)

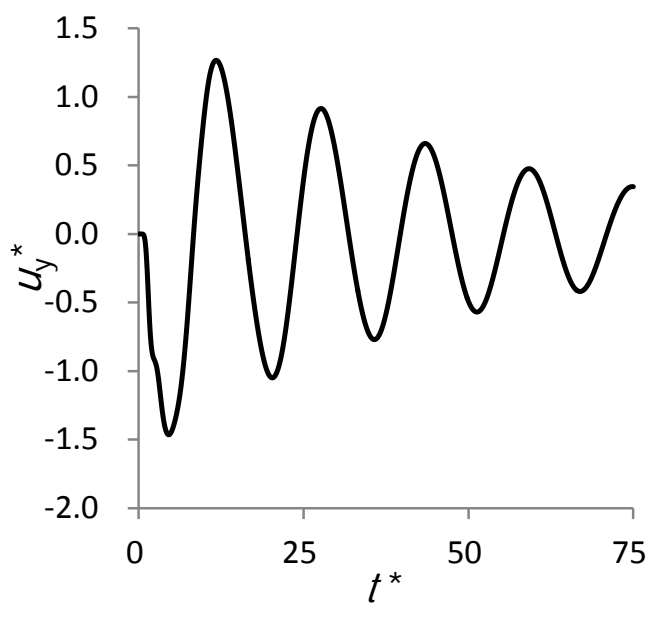

(b)

Figure 11. SBFEM transient analysis of an $L$-shaped panel: (a) pressure variation with respect to time and (b) dimensionless displacement in the $y$ direction of point $A$

The dimensionless displacement history of point $A$ (refer to Figure 9 (a)) in the $y$ direction is shown in Figure 11 (b). With reference length $r_{\mathrm{r}}=1 \mathrm{~m}$, the dimensional displacement should hold the same amplitude as $u_{y}{ }^{*}$, whereas the time variable should be calculated by reformulating equation (35) in terms of $t$ to obtain its dimensional counterpart.

An equivalent FEM analysis is also carried out for comparison purposes. Excellent agreement between FEM and SBFEM results is observed in Figure 12, which compares the displacement in the $x$ and $y$ directions of point $O$, and the displacement histories in the $y$ direction of points $C$ and $D$ (refer to Figure 9 (a)) from both FEM and SBFEM calculations. An attempt of using original parameters in the SBFEM analysis does not produce any realistic results.

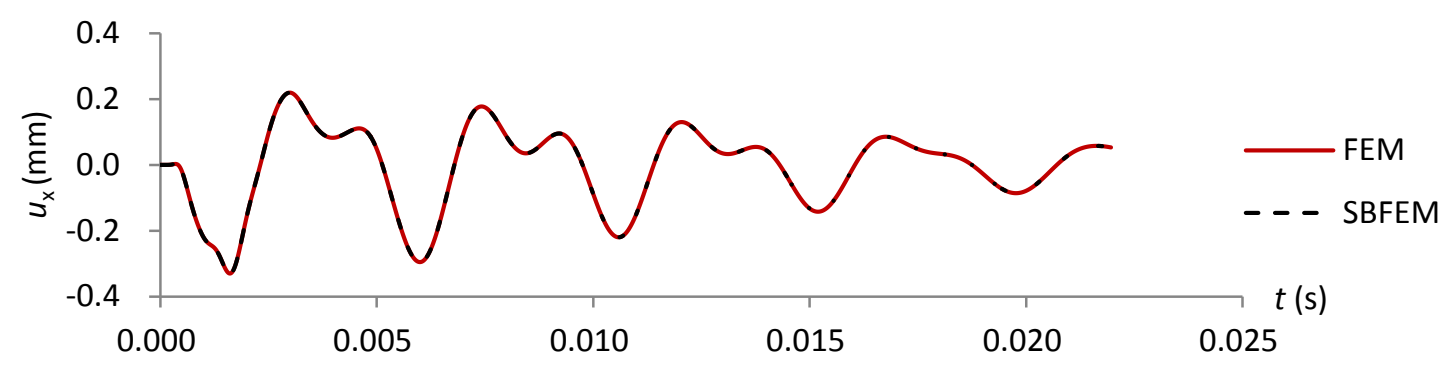

(a) 


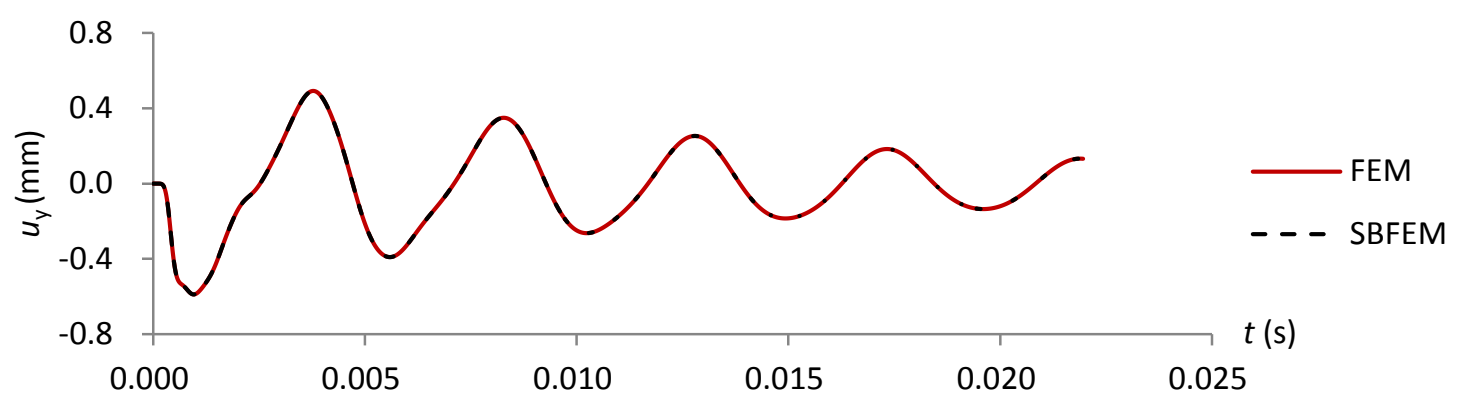

(b)

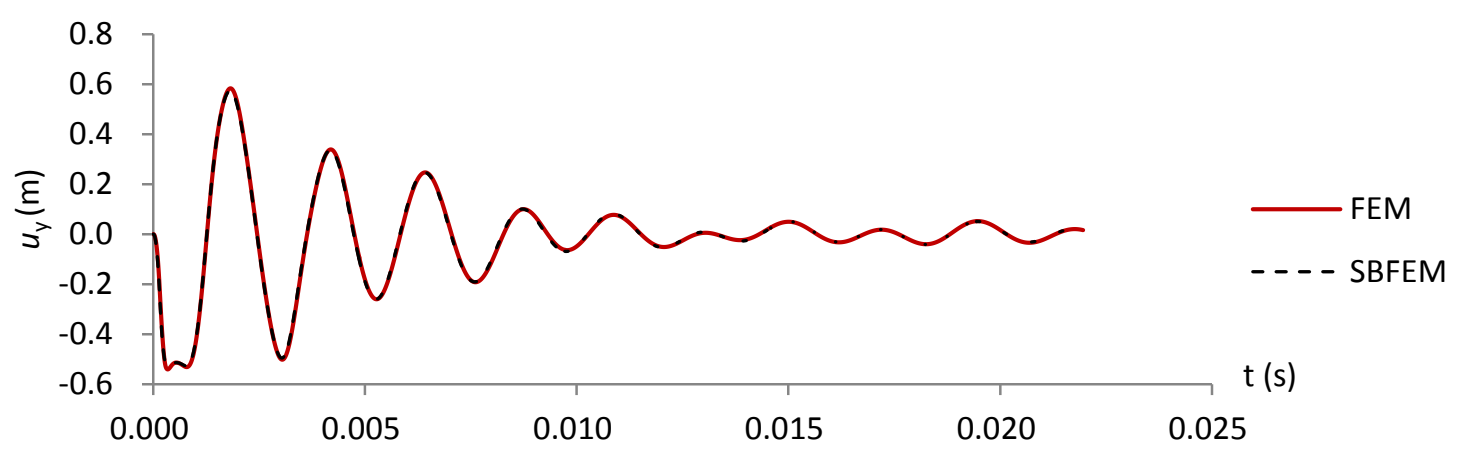

(c)

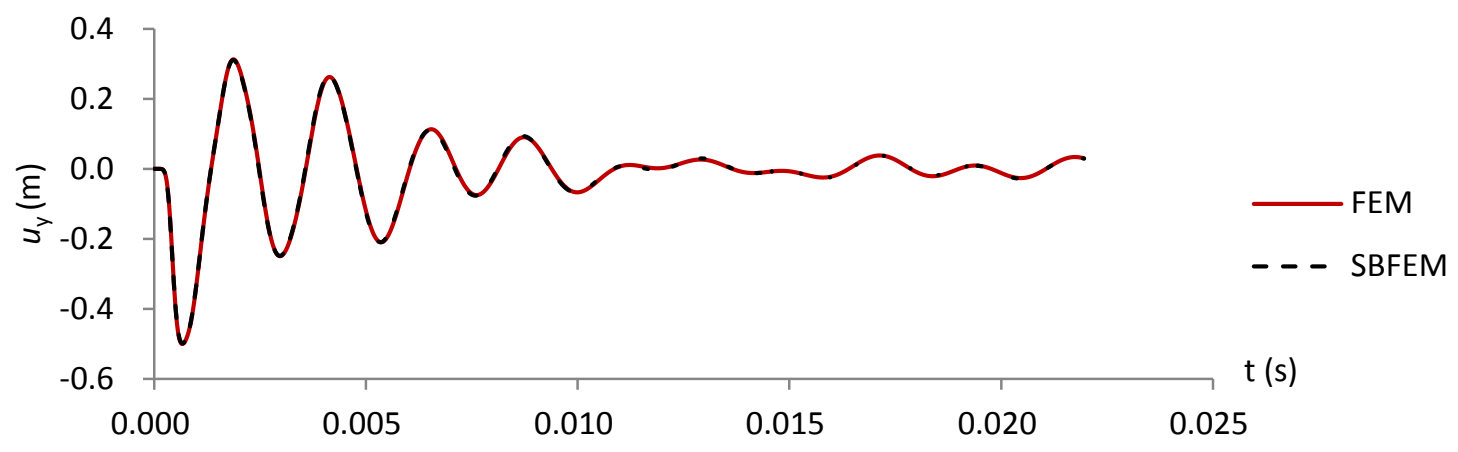

(d)

Figure 12. Comparison of displacement history between SBFEM and FEM models: (a) in the $x$ direction of point $O$; (b) in the $y$ direction of point $O$; (c) in the $y$ direction of point $C$ and (d) in the $y$ direction of point $D$

\subsubsection{Wave propagation in unbounded domains}

Revisiting the example of elastic wave propagation in an unbounded domain illustrated in Section 4.2, by applying the non-dimensionalisation process with reference parameters 
chosen as $r_{\mathrm{r}}=1 \mathrm{~m}, G_{\mathrm{r}}=1 \times 10^{10} \mathrm{~Pa}$ and $\rho_{\mathrm{r}}=2500 \mathrm{~kg} / \mathrm{m}^{3}$, the degree of difference in the magnitude of matrix entries is significantly mitigated. As shown in Table 3, for a continued-fraction order $M_{c f}$ up to 8 , the variation in the magnitude of matrix elements is within $1 \times 10^{0}$ and $1 \times 10^{10}$ as opposed to $1 \times 10^{-7}$ and $1 \times 10^{17}$ when using dimensional parameters. This suggests that when formulating the coefficient matrix $[A]$ according to equation (29), the difference in the magnitude between the largest and the smallest elements in $[A]$ is reduced from $10^{24}$ to $10^{10}$. The component of the dynamic stiffness matrix in the $z$ direction of the unbounded domain calculated at specified frequencies before and after applying the non-dimensionalisation scheme is compared in Figure 13. It is noticed that using dimensional parameters, the dynamic stiffness matrix is substantially different from that calculated using dimensionless parameters. Due to the unfavoured matrix properties, subsequent calculations from using dimensional parameters potentially lead to erroneous results, or even terminate the calculation in the process of matrix manipulations. By improving the properties of coefficient matrices, the nondimensionalisation scheme undoubtedly enhances the credibility of SBFEM calculations.

Table 3. Comparison of matrix entry magnitudes before and after applying the nondimensionalisation scheme

\begin{tabular}{c|ccc|ccc}
\hline Matrices & \multicolumn{2}{|c|}{ Before non-dimensionalisation } & \multicolumn{3}{|c}{ After non-dimensionalisation } \\
\hline$/$ & $x$ & $y$ & $z$ & $x$ & $y$ & $z$ \\
{$\left[K_{\infty}\right]$} & $4.52 \times 10^{10}$ & $4.52 \times 10^{10}$ & $6.21 \times 10^{10}$ & $4.52 \times 10^{0}$ & $4.52 \times 10^{0}$ & $6.21 \times 10^{0}$ \\
{$\left[\mathbf{Y}_{0}^{(1)}\right]$} & $2.15 \times 10^{-7}$ & $2.15 \times 10^{-7}$ & $2.54 \times 10^{-6}$ & $2.15 \times 10^{3}$ & $2.15 \times 10^{3}$ & $2.54 \times 10^{4}$ \\
{$\left[\mathbf{Y}_{0}^{(2)}\right]$} & $7.71 \times 10^{10}$ & $7.71 \times 10^{10}$ & $7.22 \times 10^{11}$ & $7.71 \times 10^{0}$ & $7.71 \times 10^{0}$ & $7.22 \times 10^{1}$ \\
{$\left[\mathbf{Y}_{0}^{(3)}\right]$} & $-1.51 \times 10^{-4}$ & $-1.51 \times 10^{-4}$ & $-5.19 \times 10^{-3}$ & $-1.51 \times 10^{6}$ & $-1.51 \times 10^{6}$ & $-5.19 \times 10^{7}$ \\
{$\left[\mathbf{Y}_{0}^{(4)}\right]$} & $-2.93 \times 10^{14}$ & $-2.75 \times 10^{14}$ & $-5.92 \times 10^{15}$ & $-1.31 \times 10^{4}$ & $-1.35 \times 10^{4}$ & $-2.92 \times 10^{4}$ \\
{$\left[\mathbf{Y}_{0}^{(5)}\right]$} & $1.55 \times 10^{-3}$ & $-1.24 \times 10^{-3}$ & $2.06 \times 10^{-1}$ & $3.03 \times 10^{9}$ & $1.14 \times 10^{9}$ & $4.82 \times 10^{9}$ \\
{$\left[\mathbf{Y}_{0}^{(6)}\right]$} & $-4.91 \times 10^{17}$ & $-4.06 \times 10^{17}$ & $-2.87 \times 10^{17}$ & $2.79 \times 10^{7}$ & $9.48 \times 10^{5}$ & $-1.99 \times 10^{6}$ \\
{$\left[\mathbf{Y}_{0}^{(7)}\right]$} & $3.06 \times 10^{-5}$ & $-1.26 \times 10^{-5}$ & $2.73 \times 10^{-4}$ & $5.02 \times 10^{8}$ & $6.25 \times 10^{8}$ & $-1.42 \times 10^{10}$
\end{tabular}




\begin{tabular}{l|lll|lll}
{$\left[\mathbf{Y}_{0}^{(8)}\right]$} & $-6.98 \times 10^{16}$ & $2.02 \times 10^{17}$ & $-5.57 \times 10^{16}$ & $7.62 \times 10^{3}$ & $7.04 \times 10^{3}$ & $5.17 \times 10^{4}$ \\
{$\left[\mathbf{Y}_{0}^{(8)}\right]$} & $1.47 \times 10^{-7}$ & $2.45 \times 10^{-7}$ & $6.14 \times 10^{-6}$ & $-2.65 \times 10^{10}$ & $-1.26 \times 10^{11}$ & $1.17 \times 10^{9}$ \\
\hline
\end{tabular}

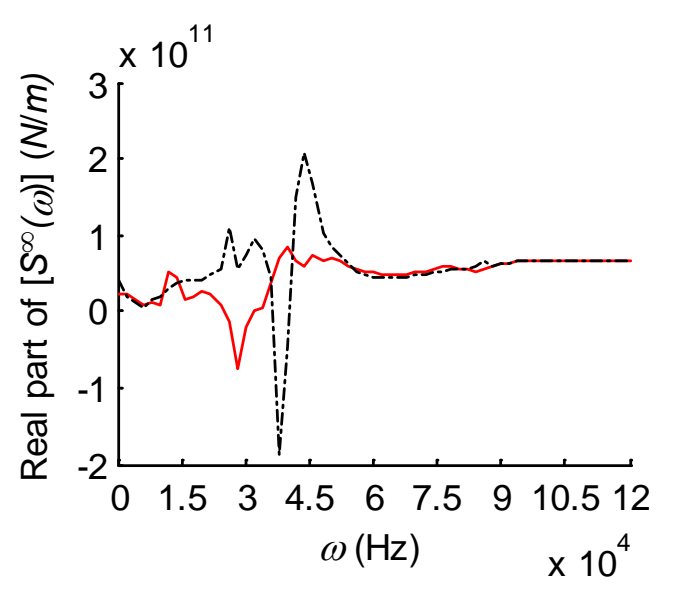

(a)

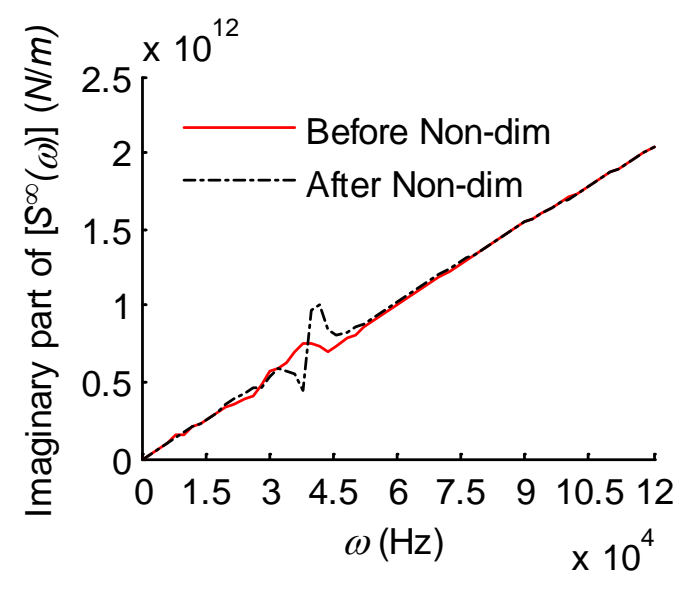

(b)

Figure 13. Comparison of the vertical dynamic stiffness coefficient before and after applying the non-dimensionalisation scheme of an elastic wave propagation problem in an unbounded domain: (a) real part of $\left[S^{\infty}(\omega)\right] v s$ frequency and (b) imaginary part of $\left[S^{\infty}(\omega)\right] v s$ frequency

\section{Conclusions}

The intense matrix calculations involved in SBFEM result in numerical instability when using this method to solve engineering problems. Therefore, in this study, emphasis is directed to the discussion of the numerical performance of SBFEM, which has not been systematically addressed in the literature. The discussion is carried out in two aspects, namely the matrix manipulation technique and the matrix properties. The eigenvalue decomposition of the Hamiltonian matrix leads to the underlying multiple eigenvalues associated with possible logarithmic terms in the solution. The real Schur decomposition can be adopted as an alternative since it circumvents this problem and provides more stable and accurate solutions. Furthermore, no manipulation of complex numbers is 
required as in the eigenvalue decomposition. A case study of a cylindrical pile subjected to uniformly distributed pressure along the circumferential direction, shows better performance of the real Schur decomposition than the eigenvalue decomposition.

On the other hand, as SBFEM relies on intensive matrix computations, the property of all relevant matrices is of significant importance to the stability and accuracy of the results. Therefore, we propose that, in all circumstances, a group of reference variables be predefined to non-dimensionalise input parameters, such as the geometric dimension, material properties and temporal variables, before performing the SBFEM calculation. All relevant matrices thus present favourable properties to ensure the correctness of the calculations. Numerical examples with respect to elasto-statics, modal and transient analyses, as well as the wave propagation problem in unbounded domain formulated using the continued-fraction technique, show enhanced performance of SBFEM after applying the proposed non-dimensionalisation scheme. This study clarifies the reasons for potential numerical instability and inaccuracy in SBFEM, with corresponding solution schemes proposed to rectify these issues. This study is expected to warranty a reliable implement of SBFEM in solving engineering problems.

\section{References}

[1] M. H. Bazyar and C. M. Song, "A continued-fraction-based high-order transmitting boundary for wave propagation in unbounded domains of arbitrary geometry", Int J Numer Meth Eng, 74 (2008) 209-237; doi: $10.1002 /$ nme.2147.

[2] C. Birk, S. Prempramote and C. Song, "An improved continued-fraction-based high-order transmitting boundary for time-domain analyses in unbounded domains", Int J Numer Meth Eng, 89 (2012) 269-298; doi: 10.1002/nme.3238.

[3] D. H. Chen, "Logarithmic singular stress field in a semi-infinite plate consisting of two edgebonded wedges subjected to surface tractions", International Journal of Fracture, 75 (1996) 357-378; doi: $10.1007 / \mathrm{bf00019615.}$

[4] R. W. Clough and J. Penzien, Dynamics of structures (McGraw-Hill, New York, 1975).

[5] A. J. Deeks and J. P. Wolf, "Semi-analytical elastostatic analysis of unbounded two-dimensional domains", International Journal for Numerical and Analytical Methods in Geomechanics, 26 (2002) 10311057; doi: 10.1002/Nag.232.

[6] A. J. Deeks and J. P. Wolf, "A virtual work derivation of the scaled boundary finite-element method for elastostatics", Computational Mechanics, 28 (2002) 489-504; doi: 10.1007/s00466-002-0314-2.

[7] A. J. Deeks and J. P. Wolf, "Semi-analytical solution of Laplace's equation in non-equilibrating unbounded problems", Comput Struct, 81 (2003) 1525-1537; doi: 10.1016/S0045-7949(03)00144-5. 
[8] K. S. Gadi, P. F. Joseph, N. S. Zhang and A. C. Kaya, "Thermally induced logarithmic stress singularities in a composite wedge and other anomalies", Eng Fract Mech, 65 (2000) 645-664; doi: 10.1016/S0013-7944(99)00145-9.

[9] D. Goldberg, "What every computer scientist should know about floating-point arithmetic", Association for Computing Machinery, Computing Surverys, 23 (1991) 5-48; doi: 10.1145/103162.103163.

[10] B. N. Li, "Extending the scaled boundary finite element method to wave diffraction problems", Ph.D. Thesis, The University of Western Australia, 2007.

[11] B. N. Li, L. Cheng, A. J. Deeks and M. Zhao, "A semi-analytical solution method for twodimensional Helmholtz equation", Applied Ocean Research, 28 (2006) 193-207; doi: 10.1016/j.apor.2006.06.003.

[12] M. Li, H. Song, H. Guan and H. Zhang, "Schur decomposition in the scaled boundary finite element method in elastostatics", Proceedings of the 9th World Congress on Computational Mechanics (WCCM) and 4th Asia-Pacific Congress on Computational Mechanics 2010 (APCOM), Minisymposia The Scaled Boundary Finite Element Method, Sydney, Australia, (2010).

[13] J. Liu, G. Lin, W. FM and J. Li, "The scaled boundary finite element method applied to Electromagnetic field problems", 9th World Congress on Computational Mechanics and 4th Asian Pacific Congress on Computational Mechanics, Sydney, Australia, (2010).

[14] C. Paige and C. Vanloan, "A Schur Decomposition for Hamiltonian Matrices", Linear Algebra Appl, 41 (1981) 11-32; doi: 10.1016/0024-3795(81)90086-0.

[15] G. B. Sinclair, "Logarithmic stress singularities resulting from various boundary conditions in angular corners of plates in extension", J Appl Mech-T Asme, 66 (1999) 556-560; doi: 10.1016/j.ijsolstr.2005.06.037.

[16] G. B. Sinclair, "Logarithmic stress singularities resulting from various boundary conditions in angular corners of plates under bending", J Appl Mech-T Asme, 67 (2000) 219-223; doi:

10.1115/1.2791085.

[17] C. M. Song, "A matrix function solution for the scaled boundary finite-element equation in statics", Computer Methods in Applied Mechanics and Engineering, 193 (2004) 2325-2356; doi: 10.1016/j.cma.2004.01.017.

[18] C. M. Song, "The scaled boundary finite element method in structural dynamics", Int J Numer Meth Eng, 77 (2009) 1139-1171; doi: 10.1002/Nme.2454.

[19] C. M. Song and J. P. Wolf, "Consistent infinitesimal finite-element cell method for diffusion equation in unobounded domain", Computer Methods in Applied Mechanics and Engineering, 132 (1996) 16; doi: 10.1016/0045-7825(96)01029-8.

[20] C. M. Song and J. P. Wolf, "The scaled boundary finite-element method - Alias consistent infinitesimal finite-element cell method - For elastodynamics", Computer Methods in Applied Mechanics and Engineering, 147 (1997) 329-355; doi: 10.1016/S0045-7825(97)00021-2.

[21] C. M. Song and J. P. Wolf, "The scaled boundary finite-element method: analytical solution in frequency domain", Computer Methods in Applied Mechanics and Engineering, 164 (1998) 249-264; doi: 10.1016/S0045-7825(98)00058-9.

[22] C. M. Song and J. P. Wolf, "The scaled boundary finite-element method - a primer: solution procedures", Comput Struct, 78 (2000) 211-225; doi: 10.1016/S0045-7949(00)00100-0.

[23] H. Song, L. B. Tao and S. Chakrabarti, "Modelling of water wave interaction with multiple cylinders of arbitrary shape", J Comput Phys, 229 (2010) 1498-1513; doi: 10.1016/j.jcp.2009.10.041.

[24] L. B. Tao, H. Song and S. Chakrabarti, "Scaled boundary FEM solution of short-crested wave diffraction by a vertical cylinder", Computer Methods in Applied Mechanics and Engineering, 197 (2007) 232-242; doi: 10.1016/j.cma.2007.07.025. 
[25] J. P. Wolf and C. M. Song, Finite-element modelling of unbounded media (Wiley, Chichester, 1996).

[26] J. P. Wolf and C. M. Song, "The scaled boundary finite-element method - a primer: derivations", Comput Struct, 78 (2000) 191-210; doi: 10.1016/S0045-7949(00)00099-7.

[27] J. P. Wolf and C. M. Song, "The scaled boundary finite-element method - a fundamental solutionless boundary-element method", Computer Methods in Applied Mechanics and Engineering, 190 (2001) 5551-5568; doi: 10.1016/S0045-7825(01)00183-9.

[28] Z. J. Yang, "Fully automatic modelling of mixed-mode crack propagation using scaled boundary finite element method", Eng Fract Mech, 73 (2006) 1711-1731; doi: 10.1016/j.engfracmech.2006.02.004.

[29] Z. J. Yang and A. J. Deeks, "Fully-automatic modelling of cohesive crack growth using a finite element-scaled boundary finite element coupled method", Eng Fract Mech, 74 (2007) 2547-2573; doi: 10.1016/j.engfracmech.2006.12.001.

[30] Z. J. Yang and A. J. Deeks, "Modelling cohesive crack growth using a two-step finite elementscaled boundary finite element coupled method", International Journal of Fracture, 143 (2007) 333-354; doi: 10.1007/s10704-007-9065-6. 